\title{
Thermomagnetic Power and Figure of Merit for Spin-1/2 Heisenberg Chain
}

\author{
Shunsuke Furukawa, Dai IKEDA and Kazumitsu SAKAI \\ Department of Physics, Tokyo Institute of Technology, Tokyo 152-8551, Japan
}

\begin{abstract}
Transport properties in the presence of magnetic fields are numerically studied for the spin$1 / 2$ Heisenberg XXZ chain. The breakdown of the spin-reversal symmetry due to the magnetic field induces the magnetothermal effect. In analogy with the thermoelectric effect in electron systems, the thermomagnetic power (magnetic Seebeck coefficient) is provided, and is numerically evaluated by the exact diagonalization for wide ranges of temperatures and various magnetic fields. For the antiferromagnetic regime, we find the magnetic Seebeck coefficient changes sign at certain temperatures, which is interpreted as an effect of strong correlations. We also compute the thermomagnetic figure of merit determining the efficiency of the thermomagnetic devices for cooling or power generation.
\end{abstract}

KEYWORDS: transport properties, thermal conductivity, magnetothermal effect, Seebeck coefficient, figure of merit, Heisenberg chain, quantum integrable system

\section{Introduction}

During the last two decades, strongly correlated systems with reduced dimensions have been extensively studied from both theoretical and experimental perspectives, due to their unique static or dynamical properties. Focusing our attention on the transport properties of magnetic materials described by one- or quasi onedimensional spin-1/2 magnets, we find their unconventional features induced by magnetic excitations. For instance, an unusually large spin diffusion constant has been observed in an NMR experiment for $\mathrm{Sr}_{2} \mathrm{CuO}_{3}{ }^{1}$ which is well characterized by the spin- $1 / 2$ Heisenberg chain. In addition, anomalously enhanced thermal conductivities were also measured in experiments for Heisenberg chain compounds $\mathrm{SrCuO}_{2}$ and $\mathrm{Sr}_{2} \mathrm{CuO}_{3},{ }^{2,3}$ indicating a quasi-ballistic heat transport carried by the spinon or magnon with a mean-free path much larger than the correlation lengths. ${ }^{4}$ Theoretically, these quasi-ballistic transport might be interpreted as a residual effect of integrability. In fact the energy current of a certain class of integrable systems including the spin-1/2 Heisenberg chain is written as a constant of motion, $, 5,6$ and therefore the heat transport exhibits purely ballistic behavior. $^{5-8}$ On the other hand the spin current is generally not conserved even in integrable systems, nevertheless it might have a finite overlap with the conserved quantities underlying integrability. Consequently the spin currentcurrent correlation does not decay to zero for long times, and then the spin transport is also considered to be ballistic. ${ }^{9-12}$ Although real materials such as $\mathrm{Sr}_{2} \mathrm{CuO}_{3}$ have non-integrable terms as a small perturbation, the system still exhibits the (quasi)-ballistic transport properties as already mentioned above. The integrability, however, is not a necessary condition to guarantee the existence of such ballistic transport properties. In fact, the quasi-ballistic heat transport was also observed in spinladder compounds $(\mathrm{Sr}, \mathrm{Ca}, \mathrm{La}){ }_{14} \mathrm{Cu}_{24} \mathrm{O}_{41},{ }^{13-16}$ which can no longer be explained by the integrability. The arising question of what kind of non-integrable systems do or do not keep the (quasi)-ballistic features still remains an intriguing open problem. ${ }^{11,12,17-27}$

Another crucial problem to be considered in the transport properties is effects of external fields. By analogy with the thermoelectric effect in electron systems, one might expect the existence of the magnetothermal effect in spin systems; the temperature gradient along a sample causes the magnetic field gradient. For the onedimensional spin systems without external fields, however, there is no magnetothermal effect since the system exhibits the spin-reversal symmetry. In the presence of finite magnetic fields, the situation drastically changes; the magnetothermal effect indeed arises due to vanishing of the spin-reversal symmetry. ${ }^{8,28,29}$ Thus for the complete understanding of the transport properties under finite external fields, we must take into account the magnetothermal effect correctly. Quite recently, by utilizing the phenomenological approach, the magnetic Seebeck coefficient has been analytically calculated in the massless regimes of the spin- $1 / 2$ Heisenberg XXZ chain. ${ }^{30}$ Most significant, in that work, was the prediction that the magnetic Seebeck coefficient changes sign at certain temperature and for certain interaction strengths. Unfortunately, the result is limited to the low-temperature region, due to the lack of conclusive results for the spin conductivity of the Heisenberg chain.

Motivated by this, in this paper, we will discuss the magnetothermal effect for the spin-1/2 Heisenberg XXZ chain beyond the limitation of the analytical approach. Namely using the exact diagonalization method, we evaluate the magnetic Seebeck coefficient for wide ranges of temperatures and various interaction strengths. As a consequence, we find the magnetic Seebeck coefficient changes sign for interaction strengths in the antiferromagnetic regime, when the magnitude of the magnetic field satisfies a certain condition. Moreover we evaluate the thermomagnetic figure of merit measuring the efficiency of the thermomagnetic devices.

The layout of this paper is as follows. In the subsequent section, we briefly present a general formulation of the transport properties in spin systems. The transport 
coefficients and the thermomagnetic power are described within linear response theory. In section 3 , we provide the spin and thermal currents for the spin- $1 / 2$ Heisenberg XXZ chain, and express the transport coefficients in terms of the correlation functions among the current operators. Numerical results by the exact diagonalization up to 18 sites are presented in section 4 . Section 5 is devoted to the summary and discussions. In appendix, we shortly give exact results for the free fermion (XY) model.

\section{Transport Coefficients in Spin Systems}

For later convenience, here we briefly provide a general formulation of the transport coefficients and the magnetothermal effect in spin systems.

Let us consider a system with two currents-the spin and heat currents-which flow as a result of forces. The transport coefficients relate the currents to the driving forces, i.e. the potential and temperature gradients. Phenomenologically these relations may be written in the form $^{28,29}$

$$
\left(\begin{array}{c}
\mathcal{J}_{\mathrm{S}} \\
\mathcal{J}_{\mathrm{Q}}
\end{array}\right)=\left(\begin{array}{ll}
L_{\mathrm{SS}} & L_{\mathrm{SQ}} \\
L_{\mathrm{QS}} & L_{\mathrm{QQ}}
\end{array}\right)\left(\begin{array}{c}
-\nabla \phi_{s} \\
-\nabla T / T
\end{array}\right)
$$

where $\mathcal{J}_{\mathrm{S}}, \mathcal{J}_{\mathrm{Q}}$ are the spin and heat currents, respectively, $\nabla \phi_{s}$ the potential gradient (typically the magnetic field gradient $\left.\nabla \phi_{s}=-\nabla h\right)$ and $\nabla T$ the temperature gradient. The Kubo formula explicitly gives the coefficients $L_{i j}$ $(\{i, j\} \in\{\mathrm{Q}, \mathrm{S}\})$ in terms of the correlation functions of the current operators:

$$
L_{i j}=\lim _{\substack{\omega \rightarrow 0 \\ \epsilon \rightarrow+0}} \operatorname{Re} \int_{0}^{\infty} \mathrm{d} t \mathrm{e}^{-\mathrm{i}(\omega-\mathrm{i} \epsilon) t} \int_{0}^{\beta} \mathrm{d} \lambda\left\langle\mathcal{J}_{i}(-t-\mathrm{i} \lambda) \mathcal{J}_{j}\right\rangle,
$$

where $\{i, j\} \in\{\mathrm{Q}, \mathrm{S}\}, \beta$ is the reciprocal temperature $\beta=1 / T$ and $\langle\cdots\rangle$ denotes the thermal expectation value per site. Using this relation with eq. (2.1), one obtains the transport coefficients for the spin system. The spin conductivity $\sigma$ is measured under the condition of no temperature gradient $\nabla T=0$ :

$$
\mathcal{J}_{\mathrm{S}}=\sigma\left(-\nabla \phi_{s}\right), \quad \sigma=L_{\mathrm{SS}} .
$$

On the other hand the thermal conductivity $\kappa$ is defined when there is no spin current $\mathcal{J}_{\mathrm{S}}=0$ :

$$
\mathcal{J}_{\mathrm{Q}}=\kappa(-\nabla T), \quad \kappa=\frac{1}{T}\left\{L_{\mathrm{QQ}}-\frac{L_{\mathrm{QS}}^{2}}{L_{\mathrm{SS}}}\right\} .
$$

Note that here we have imposed the Onsager relation $L_{\mathrm{SQ}}=L_{\mathrm{QS}}$.

On the analogy of the thermoelectric power (Seebeck coefficient) for electron systems, we define the thermomagnetic power (we refer to as the "magnetic" Seebeck coefficient) $S$, which should be measured under the condition $\mathcal{J}_{\mathrm{S}}=0$ :

$$
S=-\frac{\nabla \phi_{s}}{\nabla T}=\frac{1}{T} \frac{L_{\mathrm{QS}}}{L_{\mathrm{SS}}} .
$$

The (magnetic) Seebeck coefficient is a crucial criterion to clarify the types of carriers. Namely when the sign of $S$ is positive (negative), the spin and heat are dominantly carried by the carriers with up-spin (down-spin).
Finally as another important quantity which determines the efficiency of thermomagnetic devices for cooling or power generation, we define the thermomagnetic figure of merit

$$
Z T=\frac{S^{2} \sigma}{\kappa} T=\frac{L_{\mathrm{QS}}^{2}}{L_{\mathrm{QQ}} L_{\mathrm{ss}}-L_{Q S}^{2}} .
$$

\section{Current Operators in the Heisenberg chain}

Here we apply the formulae given in the preceding section to the spin-1/2 Heisenberg XXZ chain. The Hamiltonian is defined as

$$
\begin{aligned}
& \mathcal{H}=\sum_{k=1}^{L} h_{k k+1}-\frac{h}{2} \sum_{k=1}^{L} \sigma_{k}^{z}, \\
& h_{k k+1}=J\left\{\sigma_{k}^{+} \sigma_{k+1}^{-}+\sigma_{k+1}^{+} \sigma_{k}^{-}+\frac{\Delta}{2}\left(\sigma_{k}^{z} \sigma_{k+1}^{z}-1\right)\right\},
\end{aligned}
$$

where $\sigma_{k}^{ \pm}=\left(\sigma_{k}^{x} \pm \mathrm{i} \sigma_{k}^{y}\right) / 2$ and $\sigma_{k}^{a}(a \in\{x, y, z\})$ are the Pauli matrices associated with the $k$ th site of the chain. From now on, we assume $L$ is even, and impose the periodic boundary condition $\left(\sigma_{1}^{a}=\sigma_{L+1}^{a}\right)$. The coupling constants $J$ and $\Delta$ together with the magnetic field $h$ determine the ground state properties of the system $^{31}$ (see Fig. 1). Since the energy spectrum is invariant under both the transformations $\left(h, \mathcal{M}=\sum_{k} \sigma_{k}^{z} / 2\right) \leftrightarrow$ $(-h,-\mathcal{M})$ and $(J, \Delta) \leftrightarrow(-J,-\Delta)$, we assume $h>0$ and $J>0$.

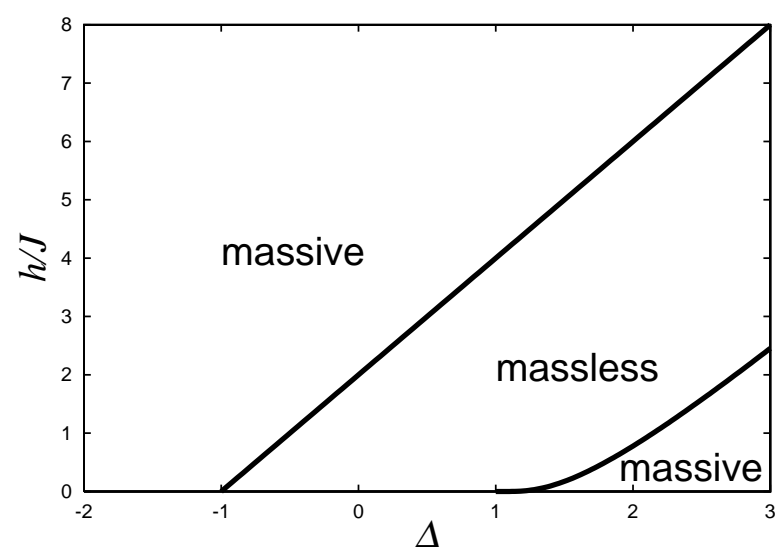

Fig. 1. Phase diagram of the ground state for $h>0$ and $J>0$.

To evaluate eq. (2.2), we define the spin and energy currents from the continuity equations for the local spin $S_{k}^{z}=\sigma_{k}^{z} / 2$ and energy $h_{k k+1}:^{5}$

$$
\dot{S}_{k}^{z}=-\operatorname{div} j_{k}^{\mathrm{S}}, \quad \dot{h}_{k k+1}=-\operatorname{div} j_{k}^{\mathrm{E}},
$$

where $\operatorname{div} j_{k}:=j_{k+1}-j_{k}$. These relations give the spin and energy current, $\mathcal{J}_{\mathrm{S}}=\sum_{k} j_{k}^{\mathrm{S}}=\sum_{k} \mathrm{i}\left[h_{k-1 k}, S_{k}^{z}\right]$ and $\mathcal{J}_{\mathrm{E}}=\sum_{k} j_{k}^{\mathrm{E}}=\sum_{k} \mathrm{i}\left[h_{k-1 k}, h_{k k+1}\right]$, respectively. Their 
explicit forms read

$$
\begin{aligned}
\mathcal{J}_{\mathrm{S}}= & \mathrm{i} J \sum_{k=1}^{L}\left(\sigma_{k}^{+} \sigma_{k+1}^{-}-\sigma_{k+1}^{+} \sigma_{k}^{-}\right) \\
\mathcal{J}_{\mathrm{E}}= & -\mathrm{i} J^{2} \sum_{k=1}^{L}\left\{\sigma_{k}^{z}\left(\sigma_{k-1}^{+} \sigma_{k+1}^{-}-\sigma_{k+1}^{+} \sigma_{k-1}^{-}\right)\right. \\
& \left.-\Delta\left(\sigma_{k-1}^{z}+\sigma_{k+2}^{z}\right)\left(\sigma_{k}^{+} \sigma_{k+1}^{-}-\sigma_{k+1}^{+} \sigma_{k}^{-}\right)\right\} .
\end{aligned}
$$

Note that the heat current $\mathcal{J}_{\mathrm{Q}}$ should be defined as

$$
\mathcal{J}_{\mathrm{Q}}=\mathrm{i} \sum\left[h_{k-1, k}-h S_{k-1}^{z}, h_{k, k+1}-h S_{k}^{z}\right]=\mathcal{J}_{\mathrm{E}}-h \mathcal{J}_{\mathrm{S}}
$$

In general, the exact evaluation of the dynamical correlation functions such as eq. (2.2) is highly challenging problem even in exactly solvable models. However, the energy current of a certain class of solvable models including the present system is written as one of the nontrivial conserved quantities underlying integrability, ${ }^{6}$ i.e. $\left[\mathcal{H}, \mathcal{J}_{\mathrm{E}}\right]=0$. This important fact directly leads to the diverging thermal conductivity at zero frequency $\omega=0$ :

$$
\kappa=\pi D_{\mathrm{Q}} \delta(\omega),
$$

where the weight of the delta function $D_{\mathrm{Q}}$ is referred to as the thermal Drude weight ${ }^{7,8,28-30}$

$$
D_{\mathrm{Q}}=\beta^{2}\left\langle\mathcal{J}_{\mathrm{E}}^{2}\right\rangle-\beta^{3} \frac{\left\langle\mathcal{J}_{\mathrm{E}} \mathcal{J}_{\mathrm{S}}\right\rangle^{2}}{D_{\mathrm{S}}}
$$

Here $D_{\mathrm{S}}$ is the Drude weight for the spin transport,

$$
\sigma=\pi D_{\mathrm{S}} \delta(\omega)+\sigma^{\mathrm{reg}}
$$

For zero magnetic field $h=0$, the magnetothermal effect, which is related to the off-diagonal dynamical correlation $L_{\mathrm{QS}}\left(=L_{\mathrm{SQ}}\right)$, is always zero (i.e. $\left\langle\mathcal{J}_{\mathrm{E}} \mathcal{J}_{\mathrm{S}}\right\rangle=0$ ) because the system exhibits the spin-reversal symmetry. In this case the thermal Drude weight is simply written as $D_{\mathrm{Q}}=\beta^{2}\left\langle\mathcal{J}_{\mathrm{E}}^{2}\right\rangle$, and can be exactly evaluated by the Bethe ansatz technique. ${ }^{7,8}$

In contrast, for finite magnetic fields $h>0$, the magnetothermal effect arises due to vanishing of the spinreversal symmetry, $\left\langle\mathcal{J}_{\mathrm{E}} \mathcal{J}_{\mathrm{S}}\right\rangle>0$. In addition to this, the spin conductivity also diverges (i.e. $D_{\mathrm{S}}>0$ ) as far as $h>0$, which is proven by using the Mazur inequality ${ }^{6}$

$$
D_{\mathrm{S}} \geq \frac{\left\langle\mathcal{J}_{\mathrm{E}} \mathcal{J}_{\mathrm{S}}\right\rangle^{2}}{T\left\langle\mathcal{J}_{\mathrm{E}}^{2}\right\rangle}>0 \quad \text { for } h>0 .
$$

Hence the thermal Drude weight (3.6) and the magnetic Seebeck coefficient (cf. (2.5))

$$
S=\frac{1}{T}\left\{\frac{\left\langle\mathcal{J}_{\mathrm{E}} \mathcal{J}_{\mathrm{S}}\right\rangle}{D_{\mathrm{S}}} \frac{1}{T}-h\right\},
$$

are both finite at finite temperatures and magnetic fields. Insertion of eqs. (3.5), (3.7) into eq. (2.6) yields

$$
Z T=\frac{S^{2} D_{\mathrm{S}}}{D_{\mathrm{Q}}} T
$$

To analyze the magnetothermal effect for the XXZ chain (3.1), we must explicitly determine the spin Drude weight as well as the correlations $\left\langle\mathcal{J}_{\mathrm{E}}^{2}\right\rangle$ and $\left\langle\mathcal{J}_{\mathrm{E}} \mathcal{J}_{\mathrm{S}}\right\rangle$. In fact, both the quantities $\left\langle\mathcal{J}_{\mathrm{E}} \mathcal{J}_{\mathrm{S}}\right\rangle$ and $\left\langle\mathcal{J}_{\mathrm{E}}^{2}\right\rangle$ have already evaluated by the Bethe ansatz in ref. 30. On the other hand, for the spin Drude weight, we may derive $D_{\mathrm{S}}$ by considering the finite size corrections of the "string" solutions to the Bethe ansatz equation. ${ }^{10,32}$ In general, however, the validity of the results obtained by applying the finite size correction to the string solutions are highly questionable under the circumstance that the breakdown of the string hypothesis is reported as in ref. 33-35. Indeed the resultant Drude weight $D_{\mathrm{S}}$ derived in ref. 10 for the case of zero magnetic field is inconsistent with that obtained by numerical analysis ${ }^{11}$ or field theoretical arguments. $^{12}$ Consequently the exact analysis of $D_{\mathrm{S}}$ at arbitrary temperatures still leaves an open problem.

Alternatively, in the next section, we will calculate the magnetic Seebeck coefficient $S$ and the figure of merit $Z T$ by the exact diagonalization for finite chains.

Finally we comment on the transport properties of the spinless fermion system given by performing the JordanWigner transformation on the XXZ chain (3.1):

$$
\begin{aligned}
\mathcal{H}_{\mathrm{c}}= & \sum_{k=1}^{L}\left\{t\left(c_{k}^{\dagger} c_{k+1}+c_{k+1}^{\dagger} c_{k}\right)+V\left(n_{k}-\frac{1}{2}\right)\left(n_{k+1}-\frac{1}{2}\right)\right\} \\
& \quad-\mu \sum_{k=1}^{L}\left(n_{k}-\frac{1}{2}\right),
\end{aligned}
$$

where $c_{k}^{\dagger}\left(c_{k}\right)$ is the fermionic creation (annihilation) operator on the site $k$ and $n_{k}=c_{k}^{\dagger} c_{k}$. Note that we have also transformed $J \rightarrow t, h \rightarrow \mu$ and $\Delta \rightarrow V /(2 t)$. The corresponding charge and energy currents are respectively given by

$$
\begin{aligned}
\mathcal{J}_{\mathrm{c}}= & e t \sum_{k=1}^{L}\left(\mathrm{i} c_{k}^{\dagger} c_{k+1}+\text { h.c. }\right) \\
\mathcal{J}_{\mathrm{E}}= & t \sum_{k=1}^{L}\left\{t\left(\mathrm{i} c_{k-1}^{\dagger} c_{k+1}+\text { h.c. }\right)\right. \\
& \left.+V\left(n_{k+1}+n_{k+2}-1\right)\left(\mathrm{i} c_{k}^{\dagger} c_{k+1}+\text { h.c. }\right)\right\},
\end{aligned}
$$

where $e$ is the charge of the particle. Accordingly the heat current is defined as $\mathcal{J}_{\mathrm{Q}}=\mathcal{J}_{\mathrm{E}}-(\mu / e) \mathcal{J}_{\mathrm{c}}(\mathrm{cf} .(3.4))$. In the thermodynamic limit $L \rightarrow \infty$ where the difference of boundary conditions between the spin system and the corresponding fermion system vanishes, the charge (thermal) conductivity $\sigma_{\mathrm{c}}\left(\kappa_{\mathrm{c}}\right)$, the Seebeck coefficient $S_{\mathrm{c}}$ and the thermoelectric figure of merit $Z_{\mathrm{c}} T$ are respectively related to those of the spin system:

$$
\sigma_{\mathrm{c}}=e^{2} \sigma, \quad \kappa_{\mathrm{c}}=\kappa, \quad S_{\mathrm{c}}=\frac{S}{e}, \quad Z_{\mathrm{c}} T=Z T .
$$

\section{Numerical Analysis for magnetic Seebeck Co- efficients and Figures of Merit}

In this section we evaluate the magnetic Seebeck coefficient $S$ and the thermomagnetic figure of merit $Z T$ by the exact diagonalization up to 18 sites.

Before we analyze them in detail, let us shortly review the magnetothermal effect for several special cases, i.e. the $\mathrm{XY}(\Delta=0)$ limit, and the low-temperature limits, where the analytical solutions are available. 


\section{$4.1 X Y$ model}

For the XY model, both the spin and thermal currents are conserved and hence the magnetothermal effect is exactly calculated by using the Jordan-Wigner transformation (see Appendix). In Fig. 2, the magnetic Seebeck coefficient $S$ together with the thermomagnetic figure of merit $Z T$ are depicted for various magnetic fields. In this case, one finds the magnetic Seebeck coefficients are always negative $(S<0)$ at any finite temperatures, which implies the carriers of the transport are dominated by the down spins.

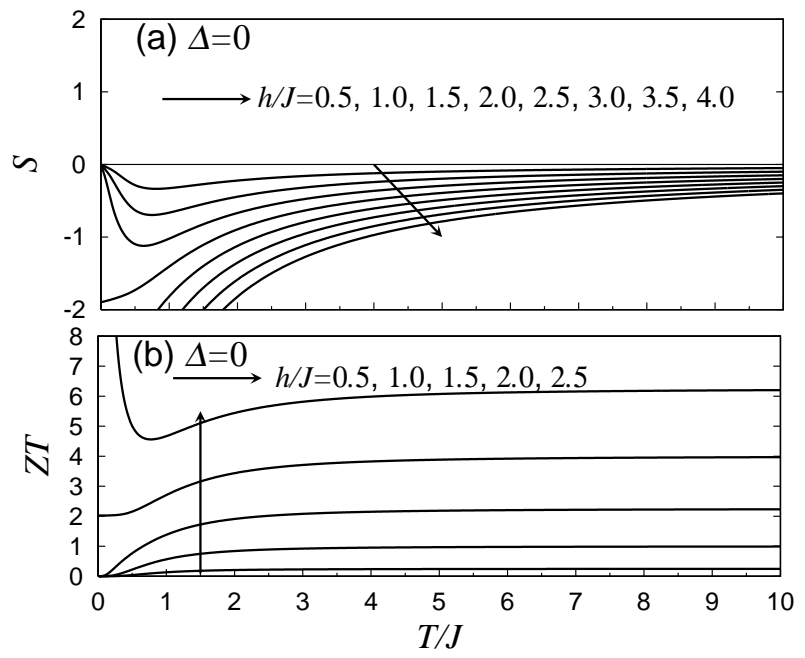

Fig. 2. Magnetic Seebeck coefficient $S$ (a) and figure of merit $Z T$ (b) for the XY model $\Delta=0$. For $h=h_{c}=2 J, S$ and $Z T$ converge to $S=-1.89738$ and $Z T=2.02697$, respectively (see eq. (A.5)).

The magnetic Seebeck coefficient $S$ for $h<h_{c}=2 J$ is linear in $T$ at low temperature. One also observes that $S$ diverges at $T=0$ as $S \sim$ const. $/ T$ (see eq. (A.5) in detail) for $h>h_{c}$, due to the mass-gap (spin insulator). Correspondingly the thermomagnetic figure of merit $Z T$ increases with increasing the magnetic fields and diverges as $Z T \sim$ const. $/ T^{2}$ at $T=0$ for $h>h_{c}$.

\subsection{Low-temperature asymptotics for $-1<\Delta \leq 1$}

Next we mention the low-temperature behavior $T \ll$ $h<h_{c}$ in the presence of the interaction strengths $-1<$ $\Delta \leq 1$. The phenomenological relation yields the leading low-temperature magnetic Seebeck coefficient for $T \ll$ $h<h_{c}: 30$

$$
S=\alpha(h) T+O\left(T^{2}\right)
$$

In Fig. 3, the coefficient $\alpha(h)$ is depicted as a function of the magnetic field for various anisotropy parameters. For weak interaction strengths $\Delta \lesssim 0.5$, the leading behavior is negative. On the contrary, for $\Delta \gtrsim 0.5$, the low-temperature behavior changes sign at certain magnetic field $h=h_{1}$ (see the inset in Fig. 3). The value $h_{1}$ shifts to higher values with the increase of the interaction strength. On the other hand, the magnetic Seebeck coefficient at high temperatures is determined according

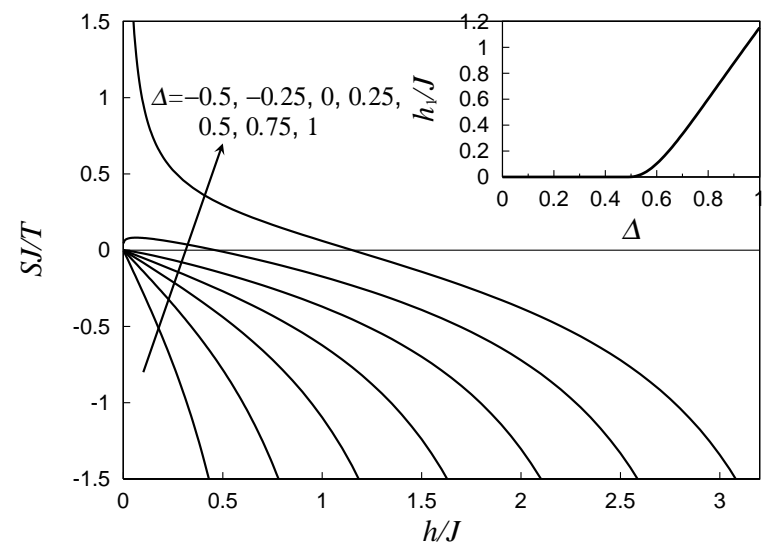

Fig. 3. Low-temperature behavior of the magnetic Seebeck coefficient $S$ in units of $T / J$ for critical regime $-1<\Delta \leq 1$. Inset: The magnetic field $h_{1}$ where the low-temperature asymptotics changes sign is shown as the function of $\Delta$.

to the argument as in ref. 36 :

$$
S=-\frac{h}{T} \quad \text { for } T \gg J .
$$

From this relation and the results in Fig. 3, we expect $S$ has a positive peak and changes sign at certain temperature $T_{0}$ at least for $\Delta \gtrsim 0.5$ and $h<h_{1}$. Namely the crossover from the regime dominated by the "downspin"-like carriers to the regime dominated by the "upspin"-like carriers occurs at $T_{0}$.

In the next subsection, beyond the limitation of the analytic methods, we numerically calculate the temperature dependence of the magnetic Seebeck coefficients for various magnetic fields and anisotropies.

\subsection{Exact diagonalization}

We next present our numerical results obtained by the exact diagonalization (ED) method. Using the conservation of the total magnetization $\mathcal{M}$ and the translational invariance of the system, we performed a full diagonalization up to $L=18$. To this end, we reduced the integral representation of the spin Drude weight $D_{\mathrm{S}}$ in (3.7) (see also (2.3) and (2.2)) to a useful form. The spectral decomposition $\mathcal{H}|m\rangle=E_{m}|m\rangle$ directly yields

$$
D_{\mathrm{S}}^{\mathrm{I}}=\beta \sum_{E_{m}=E_{n}} p_{m}\left|\left\langle m\left|\mathcal{J}_{\mathrm{S}}\right| n\right\rangle\right|^{2}, \quad p_{m}=\frac{1}{L} \frac{\mathrm{e}^{-\beta E_{m}}}{\sum_{n} \mathrm{e}^{-\beta E_{n}}}
$$

Applying the partial integration to $L_{\mathrm{SS}}(2.2)$, one also derives

$$
D_{\mathrm{S}}^{\mathrm{II}}=\langle-K\rangle-2 \sum_{E_{m} \neq E_{n}} p_{n} \frac{\left|\left\langle m\left|\mathcal{J}_{\mathrm{S}}\right| n\right\rangle\right|^{2}}{E_{m}-E_{n}}
$$

where $K$ is the kinetic term $K=J \sum_{k}\left(\sigma_{k}^{+} \sigma_{k+1}^{-}+\right.$h.c. $)$. Here we have used the relation

$$
\int_{0}^{\beta}\left\langle\mathcal{J}_{\mathrm{S}}(-\mathrm{i} \lambda) \mathcal{J}_{\mathrm{S}}\right\rangle \mathrm{d} \lambda=\langle-K\rangle
$$

which is valid in the thermodynamic limit $L \rightarrow \infty$. In Fig. 4, the size dependence of the magnetic Seebeck coefficient $S^{\mathrm{I}}\left(S^{\mathrm{II}}\right)$ calculated by using $D_{\mathrm{S}}^{\mathrm{I}}\left(D_{\mathrm{S}}^{\mathrm{II}}\right) . S^{\mathrm{I}}$ and 


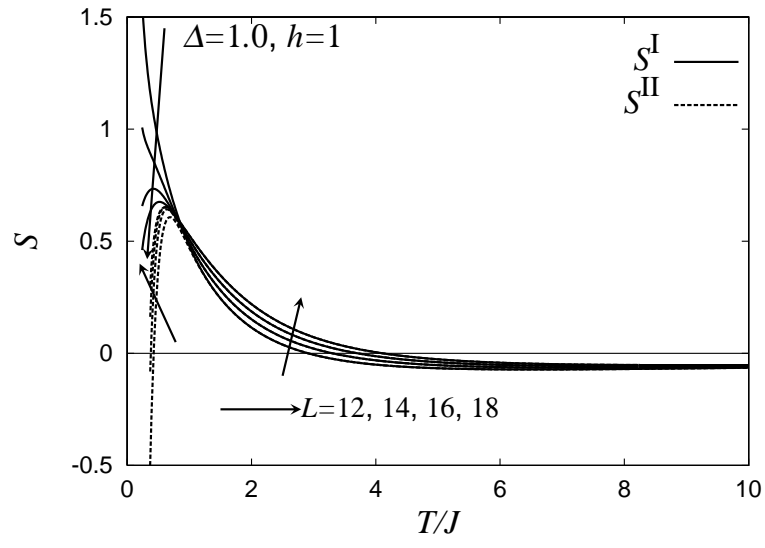

Fig. 4. Size dependence of the magnetic Seebeck coefficient $S$ for $\Delta=1$ and $h / J=1$

$S^{\mathrm{II}}$ show a good agreement at high temperatures, confirming $D_{\mathrm{S}}^{\mathrm{I}}(4.2)$ and $D_{\mathrm{S}}^{\mathrm{II}}(4.3)$ are equivalent at high temperature limit. In this region, one also observes a relatively weak size dependence. On the other hand, at low temperatures, both $S^{\mathrm{I}}$ and $S^{\mathrm{II}}$ exhibit strong size dependences, which mainly stem from the strong finite size correction of the spin Drude weight. In this region, one sees $S^{\mathrm{I}}\left(S^{\mathrm{II}}\right)$ decreases (increases) with increasing the system size. From this observation, we expect the magnetic Seebeck coefficient $S$ in the thermodynamic limit converges to an intermediate value of $S^{\mathrm{I}}$ and $S^{\mathrm{II}}$.

The magnetic Seebeck coefficient $S$ for several anisotropies $\Delta$ and magnetic fields $h$ are shown in Fig. 5 . One observes that $S$ strongly depends on $h$ and $\Delta$. As expected in eq. (4.1), $S$ is negative at high temperatures, and converges to zero at $T \rightarrow \infty$.

Above the critical field $h>h_{c}$ where all the spins point up at $T=0$, the behavior of $S$ is similar to that of the XY model. Namely $S$ monotonously decreases with increasing $h$. At low temperature regime, $S$ converges to a certain finite value for $h=h_{c}$, and diverges like $S \sim$ $-\delta / T$ for $h>h_{c}$, where $\delta$ is the one-magnon excitation gap (see later discussion).

For $h<h_{c}$, in contrast, the behavior of the magnetic Seebeck coefficients in the regime $\Delta>0$ is clearly different from that for $\Delta<0$. Namely for $\Delta>0, S$ has a positive peak (in other words $S$ changes sign) below certain magnetic field $h<h_{1}$, while for $\Delta<0, S$ is always negative as in the XY model. This behavior is consistent with the former prediction mentioned in the preceding subsection. In our investigation, however, we find that this sign changes occur even in $0<\Delta \leq 0.5$, which cannot be concluded as long as we concentrate on the low-temperature behavior since the low-temperature asymptotics is negative in this region (cf. Fig. 3). One can also find in Fig. 5 for $\Delta>0$ that the positive peak grows in height, and the temperature range where $S>0$ becomes wide with the increase of $\Delta$.

In Fig. 6 the boundary on which $S$ changes sign is shown as a line on the $h-T$ plane. The inside (outside) of the curve denotes the region $S>0(S<0)$. For any $\Delta>0$, the line has a maximum at a certain

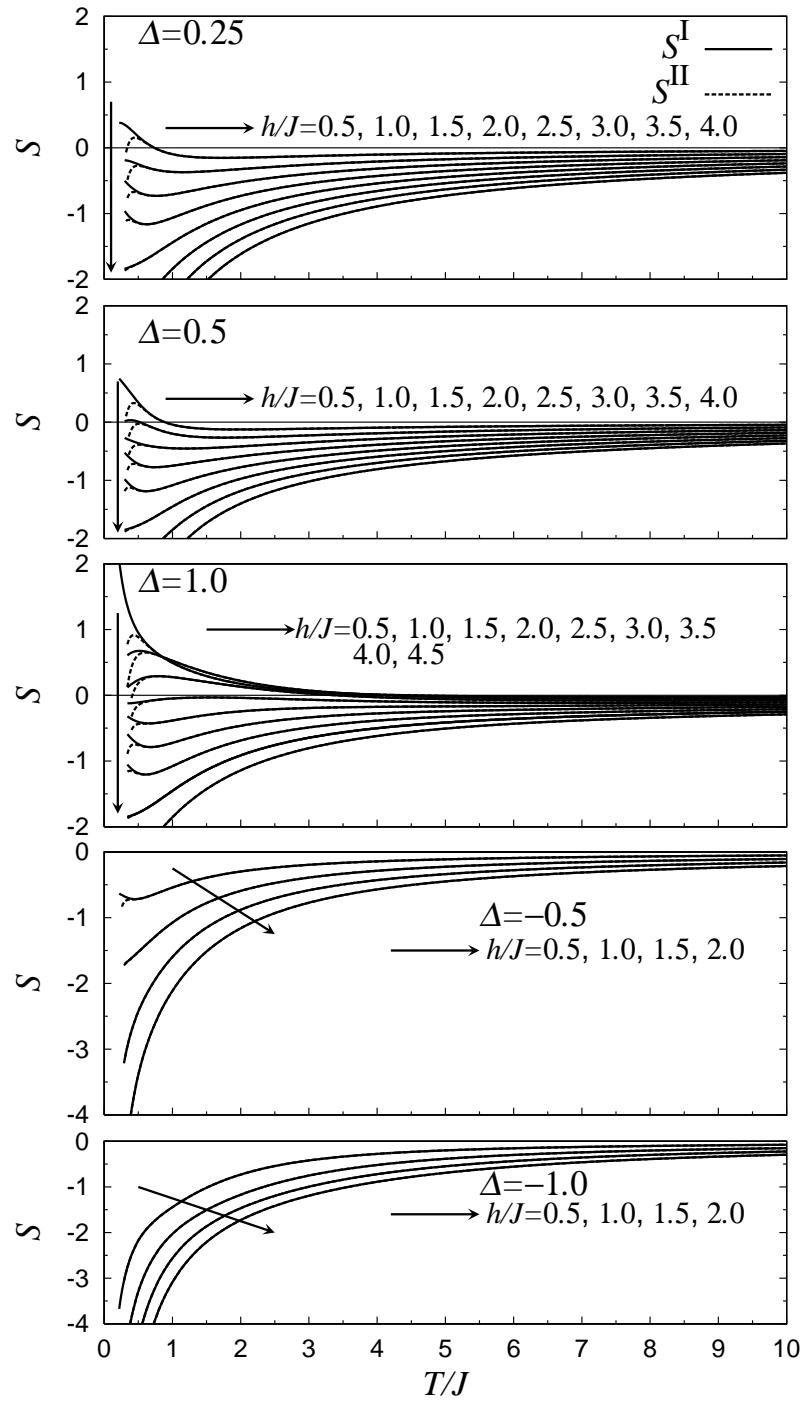

Fig. 5. Temperature dependence of the magnetic Seebeck coefficients $S$ for both $\Delta>0$ and $\Delta<0$.

finite temperature. This feature together with the lowtemperature asymptotics shown in Fig. 3 indicate that, for $h_{1}<h<h_{2}$, the sign change occurs at the temperatures $T_{1}$ and $T_{2}\left(S(T)>0\right.$ for $T_{1}<T<T_{2}$ and $S(T)<0$ for $0<T<T_{1}$ or $T>T_{2}$ ), while for $0<h<h_{1}$, it occurs at only one point $T=T_{0}\left(S(T)>0\right.$ for $T<T_{0}$ and $S(T)<0$ for $T>T_{0}$ ). Here $h_{2}$ is the height of the maximum of the line depicted in Fig. 6 and $h_{1}$ is defined in the preceding section (see Fig. 3 ).

This remarkable feature may be qualitatively interpreted by considering the following extreme cases. (i) For $h \gg 1$ where the almost every spin points up, the elementary excitation is described by the down-spin magnon. In this case, the mobility of the down-spin magnon may be larger than that of the up-spin magnon, implying the negative magnetic Seebeck coefficient $S<0$. (ii) For $\Delta \gg 1$ and $0<h \ll 1$, the excitation from the ground state is mainly characterized by the up spinon, which also plays a role as a carrier, indicating the positive magnetic Seebeck coefficient, i.e. $S>0$. (iii) For $T \gg 1$ where the interaction $\Delta$ is irrelevant, the behavior of $S$ is similar to the XY case where $S<0$. 


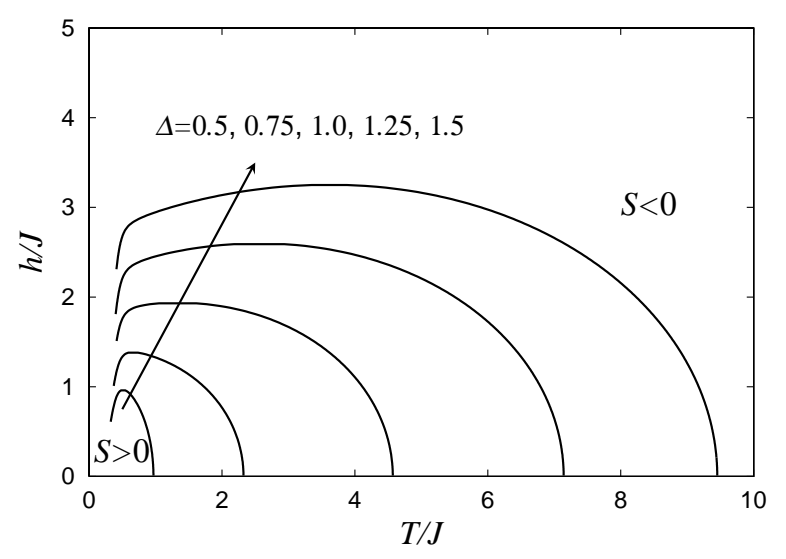

Fig. 6. Boundary where the magnetic Seebeck coefficient $S$ changes sign.

For the realistic regimes $\Delta, T, h \sim 1$, the sign changes of $S$ might be interpreted as the competition of the above three mechanisms. Namely the sign change at $T=T_{0}$ or $T=T_{2}$ is explained in terms of (ii) and (iii). On the other hand, the sign change at $T=T_{1}$ is described by (i) and (ii), i.e. the crossover from the regime dominated by the carriers of down magnons to the regime dominated by the carriers of up spinons occurs at $T=T_{1}$.

We next investigate in detail the divergent behavior of $S$ above the saturation field $h_{c}$. As shown in Fig. 7, the divergence of $S$ at low temperature can be canceled by multiplying it by $T / J$. The value of $S T / J$ approaches $-\delta / J$ in the low-temperature limit, where $\delta$ is the onemagnon excitation gap $\delta=h-2 J(\Delta+1)$. Thus we propose that the leading asymptotics at low temperature is written as $S=-\delta / T$.

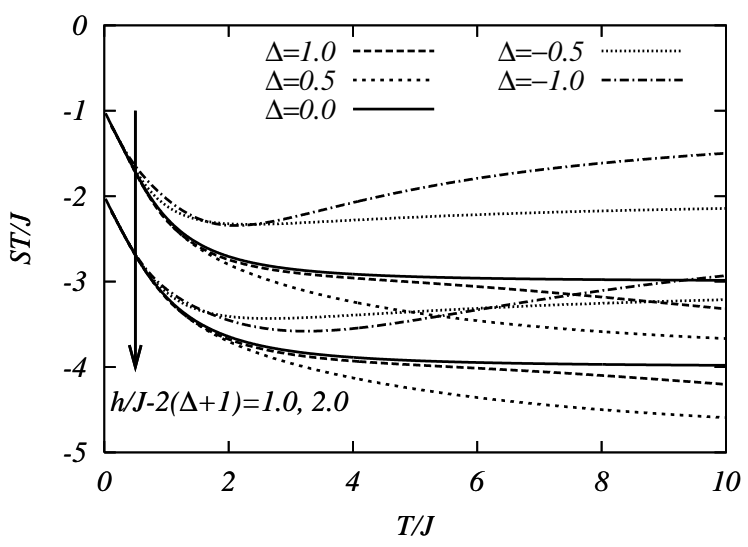

Fig. 7. The magnetic Seebeck coefficient $S$ multiplied by $T / J$ above the critical fields. In the low-temperature limit, $S$ behaves as $S \sim-\delta / T$ where $\delta$ is the one-magnon excitation gap $\delta=h-2 J(\Delta+1)$.

For $h=h_{c}$ where $S$ converges to a finite value in the low-temperature limit, we observe a universal temperature dependence of $S$ for $\Delta>0$ as shown in Fig. 8(a), i.e. the behavior of $S$ is well described by that of the noninteracting case. The existence of such a universal behav- ior at $h=h_{c}$ has also be pointed out by Heidrich-Meisner et al. in the investigation of the thermal Drude weight. ${ }^{29}$ For $\Delta<0$ in Fig. 8(b), in contrast, the temperature dependence of $S$ strongly depends on the anisotropy.

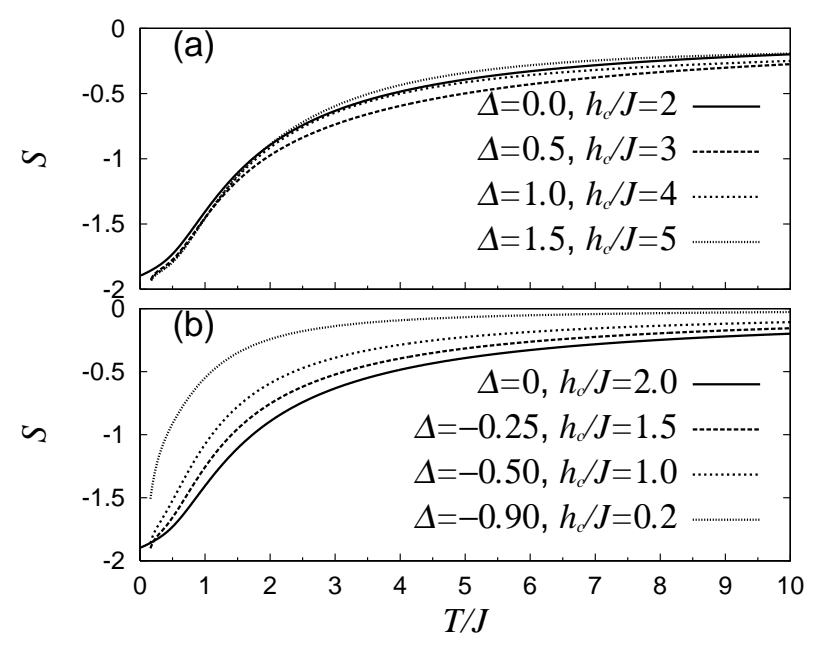

Fig. 8. Temperature dependence of the magnetic Seebeck coefficient $S$ for the critical field $h_{c}=2 J(1+\Delta)$

Finally we discuss our numerical results for the thermomagnetic figure of merit $Z T$. The temperature dependence of $Z T$ obtained by using $D_{\mathrm{S}}^{\mathrm{II}}$ is depicted in Fig. 9 for various magnetic fields and several anisotropies. For $h<h_{c}$, the figure of merit $Z T$ approaches zero at zero temperature. On the other hand, $Z T$ converges to a certain finite value for $h=h_{c}$, and diverges for $h>h_{c}$, which is similarly observed in the XY case. In general, for fixed magnetic field, $Z T$ in $\Delta>0$ tends to decrease with the increase of the interaction strength $\Delta$, reflecting the fact that the thermal Drude weight increases, while the spin Drude weight decreases with increasing $\Delta$.

\section{Summary and Discussion}

In this paper, the magnetothermal effect was studied for both the ferromagnetic and antiferromagnetic regimes of the XXZ chain with external magnetic fields. The magnetic Seebeck coefficients and the figures of merit were numerically calculated by using the exact diagonalization up to 18 sites. For the antiferromagnetic regime, we found the magnetic Seebeck coefficient changes sign at certain temperatures, which is interpreted as an effect of the competition among the interaction strengths, temperatures and external magnetic fields.

An extension to integrable correlated electron systems with the spin degree of freedom such as the Hubbard model and the supersymmetric $t-J$ model is an interesting problem. Due to the integrability, the thermal and the spin Drude weights are considered to be finite at finite temperatures. In particular, the energy current for the supersymmetric $t-J$ model is conserved and hence our method developed in this paper can be directly applied. For zero magnetic fields, Zemljic and Prelovšek have recently investigated the thermoelectric power of the Hubbard model. ${ }^{37}$ Because of strong correlations, the sign 


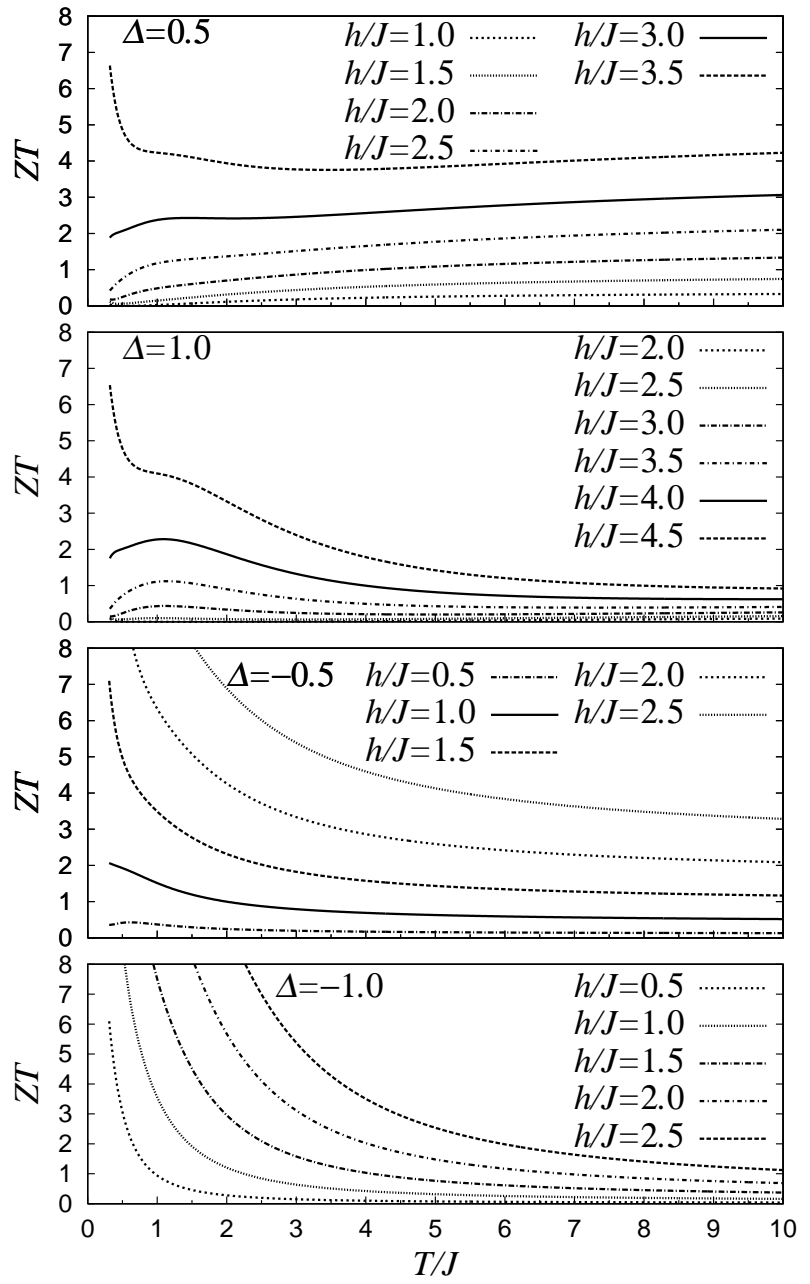

Fig. 9. Temperature dependence of the figure of merit for $Z T$ both $\Delta>0$ and $\Delta<0$.

changes of the (electric) Seebeck coefficient have been observed as in the present case. In the presence of external magnetic fields, the magnetothermal effect arises and crucially affects the charge transport and the thermoelectric effect. The investigation of this effect is also of importance for better understanding of the transport properties of strongly correlated electron systems with reduced dimensions.

\section{Acknowledgment}

We would like to thank A. Klümper and M. Oshikawa for stimulating discussions. This work is supported in part by a 21st Century COE Program at Tokyo Tech "Nanometer-Scale Quantum Physics" by the Ministry of Education, Culture, Sports, Science and Technology. KS is also supported by Grant-in-Aid for Young Scientists (B) No. 17740248.

\section{Appendix: XY Case}

In this appendix, we provide the exact results of the transport coefficients and the thermomagnetic power for the XY chain, which corresponds to $\Delta=0$ in eq. (3.1). In this case, the spin current $\mathcal{J}_{\mathrm{S}}$ is a constant of motion, i.e. $\left[\mathcal{H}, \mathcal{J}_{s}\right]=0$. Hence from eq. (4.2), one sees that the spin Drude weight is simply expressed as $D_{\mathrm{S}}=\beta\left\langle\mathcal{J}_{\mathrm{S}}^{2}\right\rangle$.
To calculate the correlation functions $\left\langle\mathcal{J}_{\mathrm{E}}^{2}\right\rangle$ and $\left\langle\mathcal{J}_{\mathrm{E}} \mathcal{J}_{\mathrm{S}}\right\rangle$, we introduce the partition function $Z$ as

$$
\ln Z\left(\lambda_{1}, \lambda_{2}\right)=\frac{\ln \operatorname{Tr} \exp \left\{-\beta \mathcal{H}+\lambda_{1} \mathcal{J}_{\mathrm{S}}+\lambda_{2} \mathcal{J}_{\mathrm{E}}\right\}}{L} .
$$

Taking the derivative of this with respect to the parameters $\lambda_{1}$ and $\lambda_{2}$, and then setting $\left(\lambda_{1}, \lambda_{2}\right)=(0,0)$, one obtains the desired quantities:

$$
\begin{aligned}
& D_{\mathrm{S}}=\beta\left\langle\mathcal{J}_{\mathrm{S}}^{2}\right\rangle=\left.\partial_{\lambda_{1}}^{2} \ln Z\left(\lambda_{1}, 0\right)\right|_{\lambda_{1}=0}, \\
& \left\langle\mathcal{J}_{\mathrm{E}}^{2}\right\rangle=\left.\partial_{\lambda_{2}}^{2} \ln Z\left(0, \lambda_{2}\right)\right|_{\lambda_{2}=0}, \\
& \left\langle\mathcal{J}_{\mathrm{E}} \mathcal{J}_{\mathrm{S}}\right\rangle=\left.\partial_{\lambda_{2}} \partial_{\lambda_{1}} \ln Z\left(\lambda_{1}, \lambda_{2}\right)\right|_{\lambda_{1}=0, \lambda_{2}=0} .
\end{aligned}
$$

For actual evaluation of $Z\left(\lambda_{1}, \lambda_{2}\right)$, it is convenient to transform the system to the spinless fermion model (3.11) (note that we set $e=1$ ). Performing the Fourier transform, one easily obtains

$$
\begin{aligned}
\mathcal{H} & =2 J \sum_{p} n_{p} \cos p-h \sum_{p}\left(n_{p}-\frac{1}{2}\right), \\
\mathcal{J}_{\mathrm{S}} & =-2 J \sum_{p} n_{p} \sin p, \quad \mathcal{J}_{\mathrm{E}}=-2 J^{2} \sum_{p} n_{p} \sin 2 p,
\end{aligned}
$$

where $p=2 \pi n / L ; n \in\{-L / 2+1,-L / 2, \cdots, L / 2-$ $1, L / 2\}$. Substituting them into $(\mathrm{A} \cdot 1)$ and taking the thermodynamic limit $L \rightarrow \infty$, we obtain

$$
\begin{aligned}
& \ln Z\left(\lambda_{1}, \lambda_{2}\right)=-\frac{\beta h}{2}+\frac{1}{2 \pi} \int_{-\pi}^{\pi} \ln \left(1+\mathrm{e}^{\varepsilon(p)}\right) \mathrm{d} p \\
& \varepsilon(p)=-2 \beta J \cos p-2 \lambda_{1} J \sin p-2 \lambda_{2} J^{2} \sin 2 p+\beta h .
\end{aligned}
$$

Thus eq. $(\mathrm{A} \cdot 2)$ yields

$$
\begin{aligned}
& D_{\mathrm{S}}=\frac{1}{2 \pi} \int_{-\pi}^{\pi} \frac{\beta J^{2} \sin ^{2} p}{\cosh ^{2}(\beta J \cos p-\beta h / 2)} \mathrm{d} p, \\
& \left\langle\mathcal{J}_{\mathrm{E}}^{2}\right\rangle=\frac{1}{2 \pi} \int_{-\pi}^{\pi} \frac{J^{4} \sin ^{2} 2 p}{\cosh ^{2}(\beta J \cos p-\beta h / 2)} \mathrm{d} p, \\
& \left\langle\mathcal{J}_{\mathrm{E}} \mathcal{J}_{\mathrm{S}}\right\rangle=\frac{1}{2 \pi} \int_{-\pi}^{\pi} \frac{J^{3} \sin p \sin 2 p}{\cosh ^{2}(\beta J \cos p-\beta h / 2)} \mathrm{d} p .
\end{aligned}
$$

Combining the above equations with (3.6), (3.9) and (3.10), one can calculate the thermal Drude weight and the magnetic Seebeck coefficient for arbitrary temperatures and magnetic fields. The low-temperature asymptotics of the magnetic Seebeck coefficient and the figure of merit are explicitly written in the following form.

$$
\begin{aligned}
& S= \begin{cases}-\frac{\pi^{2} h}{3 v_{s}^{2} \beta}+O\left(\beta^{-3}\right) & \text { for } h<h_{c} \\
-\frac{g(3)}{g(1)}+O\left(\beta^{-1}\right) & \text { for } h=h_{c} \\
(2 J-h) \beta+O(1) & \text { for } h>h_{c}\end{cases} \\
& Z T= \begin{cases}\frac{\pi^{2} h}{3 v_{s}^{2} \beta^{2}}+O\left(\beta^{-4}\right) & \text { for } h<h_{c} \\
\frac{g^{2}(3)}{g(1) g(5)-g^{2}(3)}+O\left(\beta^{-1}\right) & \text { for } h=h_{c}, \\
\frac{2}{3}(2 J-h)^{2} \beta^{2}+O(\beta) & \text { for } h>h_{c}\end{cases}
\end{aligned}
$$


where $v_{s}=\sqrt{4 J^{2}-h^{2}}$ is the velocity of excitations, $h_{c}=2 J$ and $g(n):=\left(1-2^{1-n / 2}\right) \Gamma(1+n / 2) \zeta(n / 2)$ (note that $\Gamma(x)$ is the Gamma function and $\zeta(x)$ the Riemann zeta function). On the other hand, the high-temperature asymptotics are given by

$$
S \sim-h \beta+O\left(\beta^{2}\right), \quad Z T \sim \frac{h^{2}}{J^{2}} .
$$

1) M. Takigawa, et al.: Phys. Rev. Lett. 76 (1996) 4612.

2) A. V. Sologubenko, et al.: Phys. Rev. B 62 (2000) R6108.

3) A. V. Sologubenko, et al.: Phys. Rev. B. 84 (2001) 054412.

4) A. V. Sologubenko, et al.: Europhys. Lett. 62 (2003) 540.

5) For a review see $X$. Zotos and P. Prelovšek: condmat/0304630.

6) X. Zotos, F. Naef and P. Prelovšek: Phys. Rev. B 55 (1997) 11029.

7) A. Klümper and K. Sakai: J. Phys. A 35 (2002) 2173.

8) K. Sakai and A. Klümper: J. Phys. A 36 (2003) 11617.

9) B. N. Narozhny, A. J. Millis and N. Andrei: Phys. Rev. B 58 (1998) R2921.

10) X. Zotos: Phys. Rev. Lett. 82 (1999) 1764.

11) J. V. Alvarez and C. Gros: Phys. Rev. Lett. 88 (2002) 077203.

12) S. Fujimoto and N. Kawakami: Phys. Rev. Lett. 90 (2003) 197202.

13) K. Kudo, et al.: J. Low. Temp. Phys. 117 (1999) 1689.

14) A. V. Sologubenko, et al.: Phys. Rev. Lett. 84 (2000) 2714.

15) C. Hess, et al.: Phys. Rev. B 64 (2001) 184305.
16) K. Kudo, et al.: J. Phys. Soc. Jpn. 70 (2001) 437.

17) F. Heidrich-Meisner, et al:: Phys. Rev. B 66, 140406(R) (2002).

18) J. V. Alvarez and C. Gros, Phys. Rev. Lett. 89, 156603 (2002).

19) E. Orignac, R. Chitra and R.Citro, Phys. Rev. B 67, 134426 (2003).

20) K. Saito and S. Miyashita, J. Phys. Soc. Jpn. 71, 2485 (2002).

21) K. Saito, Phys. Rev. B 67, 064410 (2003).

22) K. Saito, Phys. Rev. B 67, 164410 (2003).

23) F. Heidrich-Meisner, A. Honecker, D.C. Cabra and W. Brenig, Phys. Rev. B 68 (2003) 134436.

$24)$ E. Shimshoni, N. Andrei and A. Rosch, Phys. Rev. B 68 104401 (2003).

25) K. Louis and C. Gros, Phys. Rev. B 68 (2003) 184424.

26) X. Zotos, Phys. Rev. Lett. 92 (2004) 067202.

27) J. Karadamoglou and X. Zotos, Phys. Rev. Lett. 93 (2004) 177203

28) K. Louis and C. Gros: Phys. Rev. B 67 (2003) 224410.

29) F. Heidrich-Meisner, et al.: Phys. Rev. B 71, 184415 (2005).

30) K. Sakai, A. Klümper: J. Phys. Soc. Jpn. 74 (2005) Suppl. 196. cond-mat/0410192.

31) M. Takahashi, Thermodynamics of One-Dimensional Solvable Models, (Cambridge: Cambridge University Press).

32) S. Fujimoto and N. Kawakami, J. Phys. A 31, 465 (1998).

33) H.L. Eßler, V.E. Korepin and K. Schoutens, J. Phys. A 25 (1992) 4115

34) G. Jüttner and B.D. Dörfel, J. Phys. A 26 (1993) 3105.

35) F.C. Alcaraz and M.J. Martins, J. Phys. A21 (1988) L381, ibid. 4397.

36) P. M. Chaikin, G. Beni, Phys. Rev. B 13 (1976) 647.

37) M. M. Zemljic and P. Prelovšek: Phys. Rev. B 71 (2005) 085110 . 